\title{
Siringoma condroide maligno: a propósito de un caso
}

\section{Malignant chondroid syringoma: case report}

\author{
Casteleiro Roca, P.*, Vázquez Barro, A.**, Comellas Franco, M.**, \\ Pombo Otero, J.***, Martelo Villar, F.*****
}

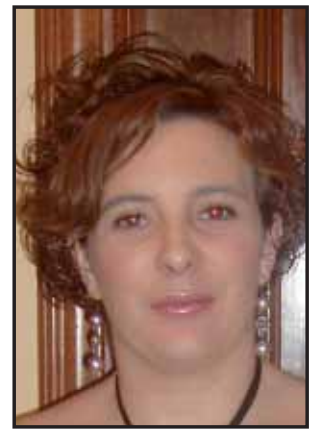

Casteleiro Roca, $\mathbf{P}$.
Resumen

El siringoma condroide maligno es un tumor muy infrecuente de origen epitelial. Presentamos el caso de una mujer de 68 años con una tumoración en su brazo izquierdo, sin invasión ósea local, pero con afectación metastásica pulmonar. El diagnóstico fue de siringoma condroide maligno. Analizamos el caso, infrecuente por el tamaño del tumor y por su evolución y hacemos una revisión bibliográfica sobre el tema.

\begin{tabular}{|c|c|}
\hline Palabras clave & $\begin{array}{l}\text { Siringoma condroide maligno. Tumores } \\
\text { cutáneos mixtos malignos. }\end{array}$ \\
\hline ilin numóni & $3-171$ \\
\hline
\end{tabular}

\begin{tabular}{|ll|}
\hline Key words & $\begin{array}{l}\text { Malignant Chondroid Syringoma. Malig- } \\
\text { nant mixed tumour of the skin. }\end{array}$ \\
Numeral Code & $3-171$
\end{tabular}

\footnotetext{
* Médico Interno Residente Cirugía Plástica.

* Médico Adjunto Cirugía Plástica.

* Médico Interno Residente Anatomía Patológica.

* J J efe de Servicio de Cirugía Plástica.

Servicio de Cirugía Plástica y Quemados. Complejo Hospitalario J uan Canalejo. La Coruña. España
}

Malignant chondroid siringoma is a rare tumour with epithelial ethilogy. We present a clinical case: a 68 years old woman with a tumour on her left arm, without local bone invasion but with metastatic injury. Diagnosis was, malignant chondroid syringoma. We present this case because of its size and evolution and a review of literature.

Abstract 


\section{Introducción}

Se denomina tumor mixto cutáneo (TMC) a aquella tumoración que presenta características epiteliales y mesenquimales de modo simultáneo, pudiendo el estroma ser mixoide, condroide, fibroso, óseo o adiposo (1). El TMC es una lesión benigna, histológica e inmnunohistoquímicamente similar en apariencia a los tumores mixtos benignos de la glándula salival (adenoma pleomórfico) (2), descrito por primera vez por Billroth como tumor mixto (3). Más de cien años después, Hirsch y Helwig describieron una formación cutánea mixta que denominaron siringoma condroide y propusieron una serie de criterios diagnósticos: tumor cutáneo o subcutáneo de pequeño tamaño, sólido, de crecimiento lento, generalmente único, cubierto de piel normal, sin molestias subjetivas, puede ser multilobulado y su localización más frecuente es en cara y cuello $(4,5)$.

El tumor cutáneo mixto benigno es relativamente frecuente, sin embargo, el siringoma condroide maligno es extremadamente raro (6). Es más común en mujeres (mujer: hombre 3:2), su localización es más frecuente en tronco y extremidades que en cabeza y cuello; frecuentemente es de mayor tamaño que la forma benigna (7), se presenta como un nódulo firme, subcutáneo, de crecimiento lento, único simple o múltiple, sin predilección por una determinada edad (8).

Presentamos un caso de siringoma condroide maligno en una mujer, atípico por su gran tamaño y por la presencia de metástasis pulmonares

\section{CASO CLÍNICO}

Mujer de 68 años de edad sin antecedentes de interés que acude a Urgencias por sangrado de una tumoración localizada en el brazo izquierdo. La tumoración es de gran tamaño $(21 \times 15 \mathrm{~cm})$ y ocupa toda la mitad distal del brazo, está ulcerada, con zonas necróticas con gran fragilidad cutánea y sangrante. Refiere haber notado una tumoración de pequeño diámetro que había ido aumentando de tamaño de modo progresivo en los últimos 2 años y por la que nunca había consultado (Fig. 1-3).

En Urgencias se procede a realizar electrocoagulación del punto sangrante bajo anestesia local y se toma una muestra del tejido para estudio anatomopatológico.

Es ingresada en el Hospital y sometida a estudio. Se le realizan pruebas preoperatorias, así como pruebas de diagnóstico por imagen y un estudio de extensión tumoral. La radiografía del brazo muestra una masa de partes blandas sin invasión ósea (Fig. 4,5). El estudio de extensión (TAC tóraco-abdomino-pélvico)
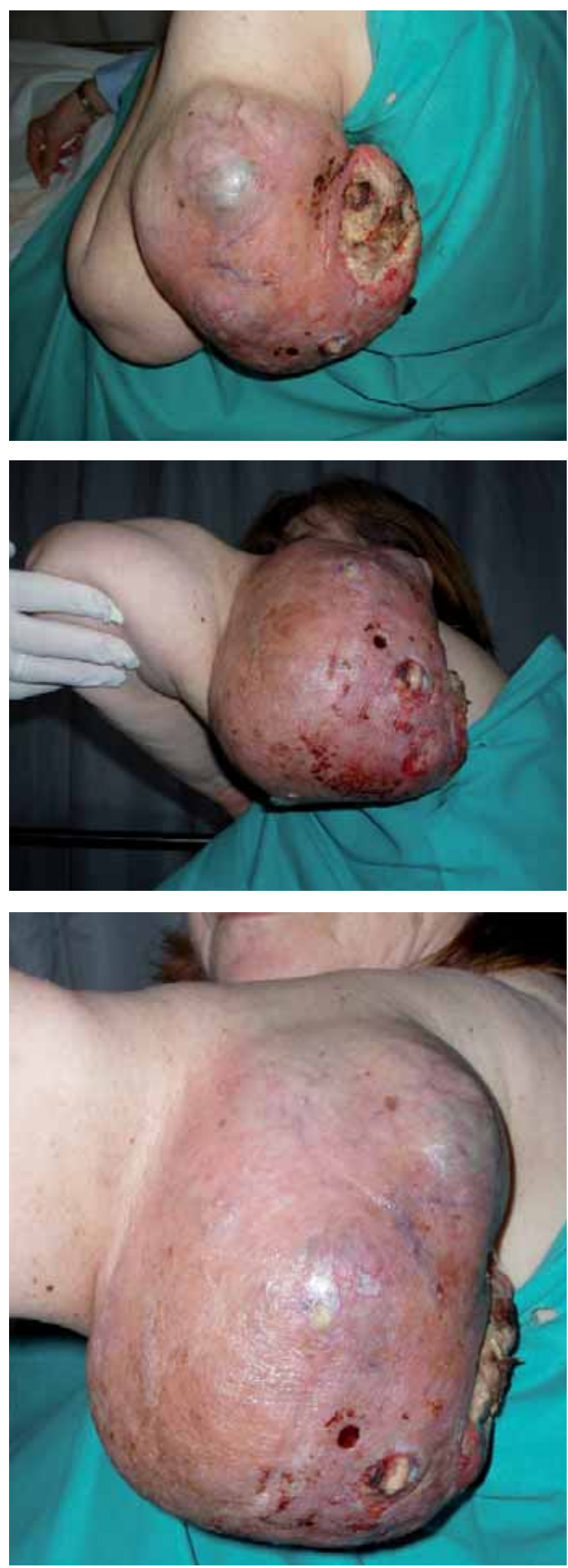

Fig. 1-3. Mujer de 68 años con siringoma condroide maligno en brazo izquierdo de $12 \times 15 \mathrm{~cm}$ de tamaño y 2 años de evolución. Imágenes preoperatorias. 

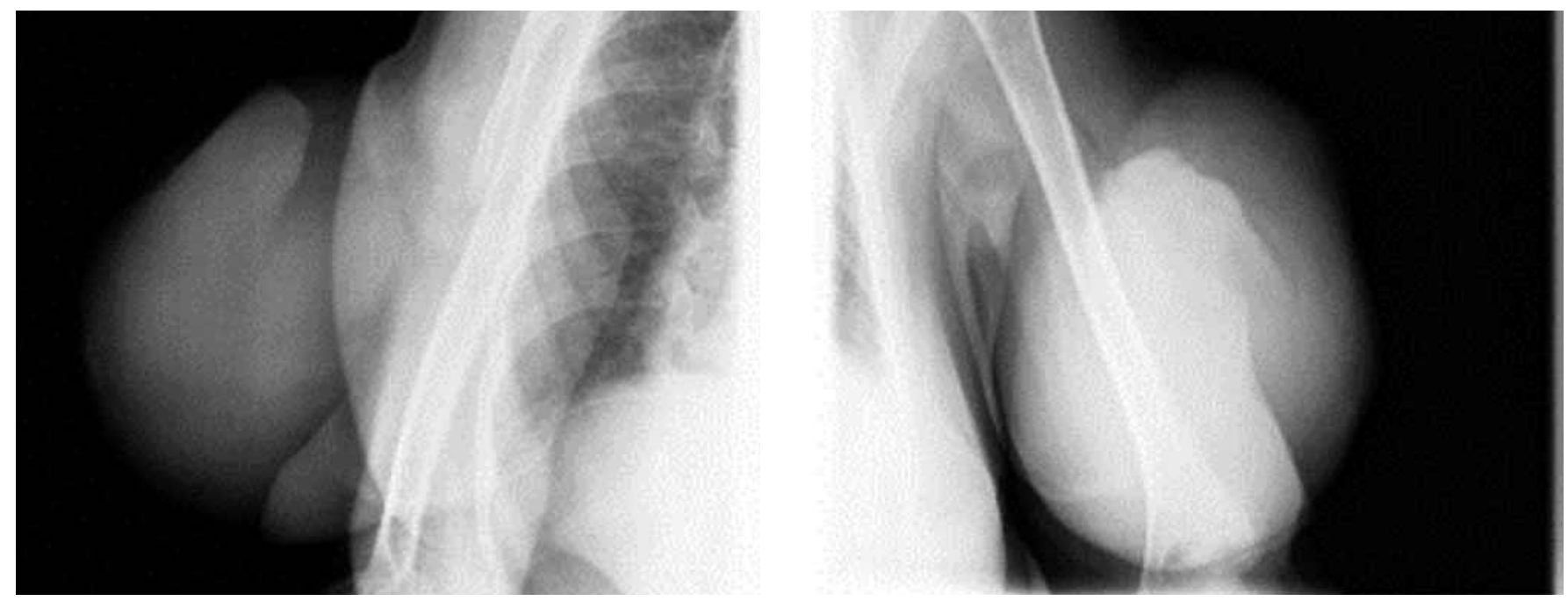

Fig. 4 y 5. Imágenes radiográficas: tumoración de partes blandas sin invasión ósea. (Imagen anterior y posterior).

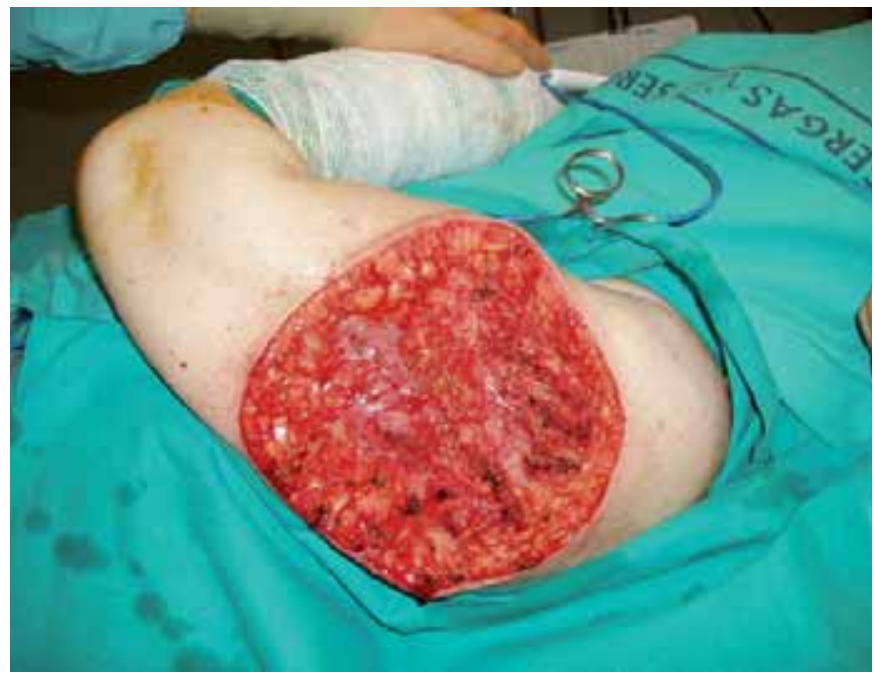

Fig. 6. Imagen intraoperatoria: Resección tumoral completa con márgenes amplios.

presenta un nódulo pulmonar de bordes bien delimitados y localización yuxtapleural, situado en el segmento superior del lóbulo inferior izquierdo, de unos $6 \mathrm{~mm}$ sugestivo de metástasis; muestra también adenopatías axilares ipsilaterales.

La biopsia de la tumoración da como resultado una zona con masiva infiltración de toda la dermis por una proliferación fibrohistiocítica que aparenta ser de bajo grado y otra zona necrosada, que sugiere una proliferación de alto grado. Se plantean como diagnósticos diferenciales tumores tipo fibrohistiocitoma maligno, sarcoma fibromixoide o mixofibrosarcoma.

Una vez completados los estudios la paciente fue valorada en sesión clínica conjunta por los Servicios de Cirugía Plástica, Oncología y Cirugía Torácica para decidir la actitud terapéutica a tomar. Se opta por tratamiento quirúrgico de la tumoración y aplicación de quimioterapia adyuvante sobre el nódulo pulmonar, que no se considera subsidiario de extirpación quirúrgica.

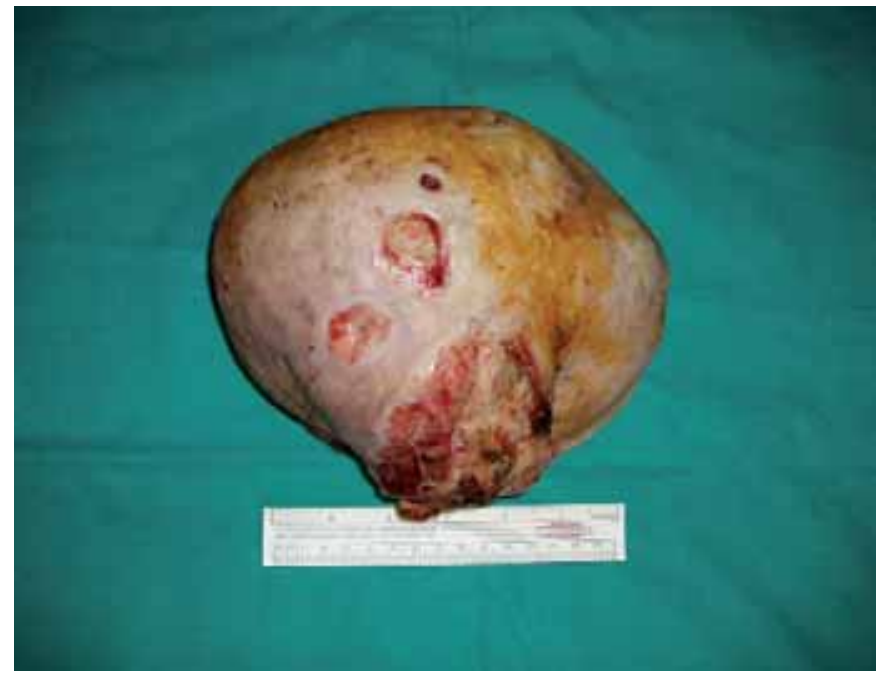

Fig. 7. Pieza de resección: 21×16x15 cm de diámetro.

La intervención consistió en la resección completa del tumor con márgenes amplios y el cierre directo del defecto subyacente (Fig. 6,7). El tratamiento quimioterápico adyuvante fue de 6 ciclos con Ifosmamida, Mesna, Adriamicina y Dacarbacina.

El estudio anatomopatológico de la tumoración extirpada reveló macroscópicamente una pieza de $21 \times 16 \times 15 \mathrm{~cm}$, parcialmente revestida por piel con importantes zonas de profunda ulceración y borde de crecimiento de tipo expansivo; al corte presentó extensas áreas centrales de necrosis, consistencia firme elástica y coloración blanquecina en las zonas no necróticas y un aspecto mixoide en otras zonas. El estudio microscópico determinó un patrón global nodular, aunque en muchas zonas estaba perdido por el gran tamaño de los nódulos. Histológicamente había aspectos muy variables, predominando las zonas de aspecto sarcomatoide con células alargadas de núcleos ovoides y citoplasma discretamente acidófilo; en otras zonas había cantidades variables de 


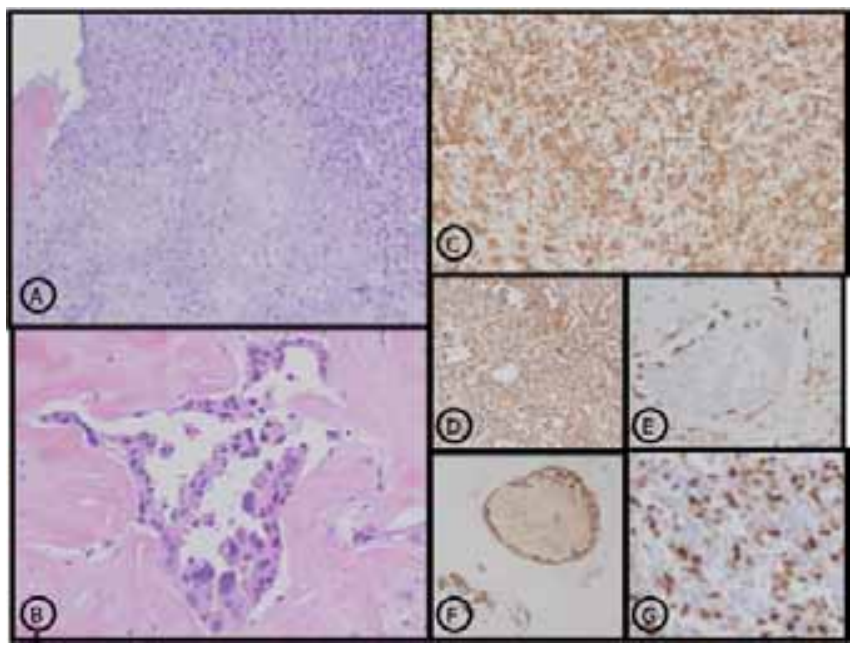

Fig. 8. Imagen anatomopatológíca de la pieza de resección: Estudio microscópico que muestra un tumor con celularidad elevada y zonas condroides (A: HEx100), otras zonas de aspecto claramente epitelial (B: HEx200). El estudio inmunohistoquímico con método de inmunoproxidasa muestra tinción positiva para S-100 y Vimentina en las zonas de aspecto mesenquimal (C y D) y negativo en las zonas epiteliales (E) mientras que las citoqueratinas (F) son positivas en las zonas epiteliales. EI índice de proliferación valorado con el MIB-I es muy alto (G).

material mucoide, áreas de predominio mixoide e incluso de claro aspecto cartilaginoso; el otro componente claramente identificado fue de tipo epitelial, glanduliforme y a menudo cilindromatoso. El estudio inmunohistoquímico fue positivo para Citoqueratinas (AE1-AE3) en las zonas epiteliales y negativo en las zonas sarcomatoides, Vimentina positivo en zonas sarcomatosas y negativo en zonas epiteliales puras, Proteína s-100 positivo en zonas sarcomatoides y negativo en las epiteliales, EMA positivo en zonas epiteliales, MIB I (índice de proliferación) elevadísimo (50\%), Actina y Desmina negativo, C-Kit negativo. El diagnóstico histológico definitivo fue de tumor de origen epitelial de alto grado de malignidad, denominado siringoma condroide maligno (Fig. 8).

La paciente fue seguida en consulta externa de Cirugía Plástica, presentando buena evolución de la cicatriz, sin evidencia de recidiva a los 2 años (Fig. 9,10). Resonancia Nuclear Magnética (RNM) del brazo sin evidencia de recidiva local. Los estudios de imagen de control tóraco-abdominales muestran disminución de las adenopatías axilares y del nódulo pulmonar $(4 \mathrm{~mm})$. Actualmente se siguen realizando controles trimestrales por parte de los Servicios de Cirugía Plástica y Oncología.

\section{Discusión}

Se han utilizado las denominaciones siringoma condroide y tumor mixto como sinónimas; si se localiza en la glándula salival se denomina adenoma pleomórfico. Es muy rara la presentación de este tipo de

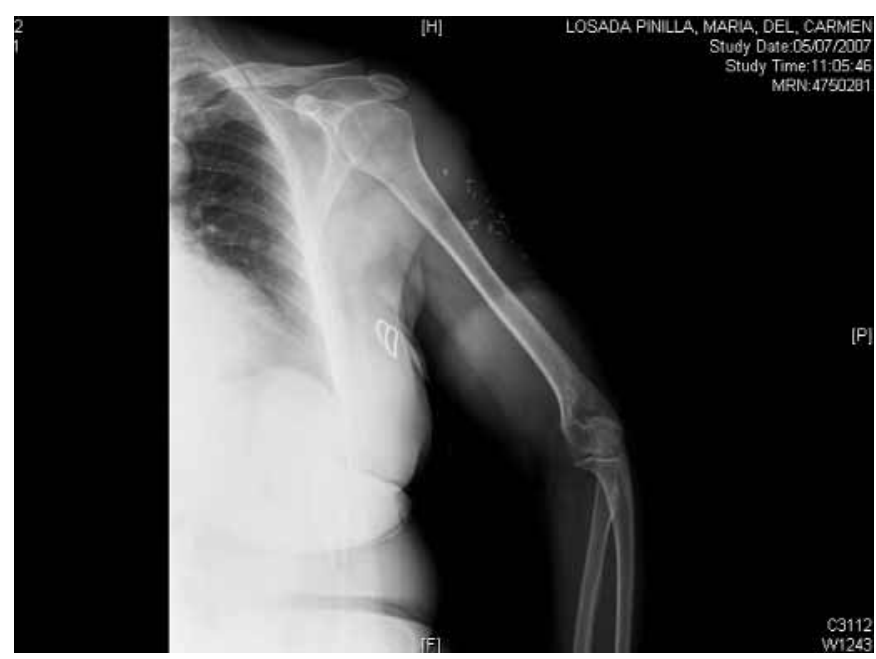

Fig. 9. Radiografía postoperatoria.

tumores fuera de las glándulas salivares, pero a veces ocurre. En 1959, Scout y Gorman revisaron la literatura, encontrando 134 casos de tumores mixtos en la piel (edad 40-60 años, sin preferencia por sexo y más frecuentes en cara y cuello) (9).

Histológicamente se trata de tumores de origen epitelial capaces de producir matriz mucoide y estructuras similares a los anejos (glándulas sudoríparas). Habría dos tipos de patrones histológicos que no se presentan nunca de forma pura, sino mezcladas. En una predominan las luces tubulares grandes y a veces quísticas, con dos capas de células, una cuboide y otra aplanada. En la segunda forma, las luces tubulares son pequeñas y están cubiertas por una sola envoltura celular de donde salen prolongaciones en forma de coma ("cola de renacuajo") (10). Inmnunohistoquímicamente, en el siringoma condroide serían positivos el GFAP, Proteína S100 y Citoqueratinas; estos tres parámetros nos ayudarían al diagnóstico (11). Histológicamente es sugestiva de malignidad la atipia celular con actividad mitótica incrementada, la presencia de márgenes infiltrados, la necrosis tumoral y la presencia de nódulos satélites (12).

Es difícil hacer el diagnóstico de siringoma condroide antes de realizar la biopsia y el resultado de la misma puede no ser definitivo puesto que se trata de un tumor compuesto por dos partes muy diferentes. El diagnóstico final lo ofrece el examen histopatológico e inmunohistoquímico de la pieza tumoral completa $(6,9)$ y se realiza por exclusión frente a otras entidades, como el mioepitelioma, el coristoma condroide, el tumor fibromixoide, el quiste sebáceo, la implantación dermoide, el neurofibroma, el dermatofibroma, el carcinoma basocelular, el carcinoma de células escamosas, pilomatrixoma, histiocitoma $(12,13)$.

Los tumores mixtos cutáneos malignos son lesiones de origen incierto, probablemente originados por la proliferación de células ectomesenquimales. La mul- 


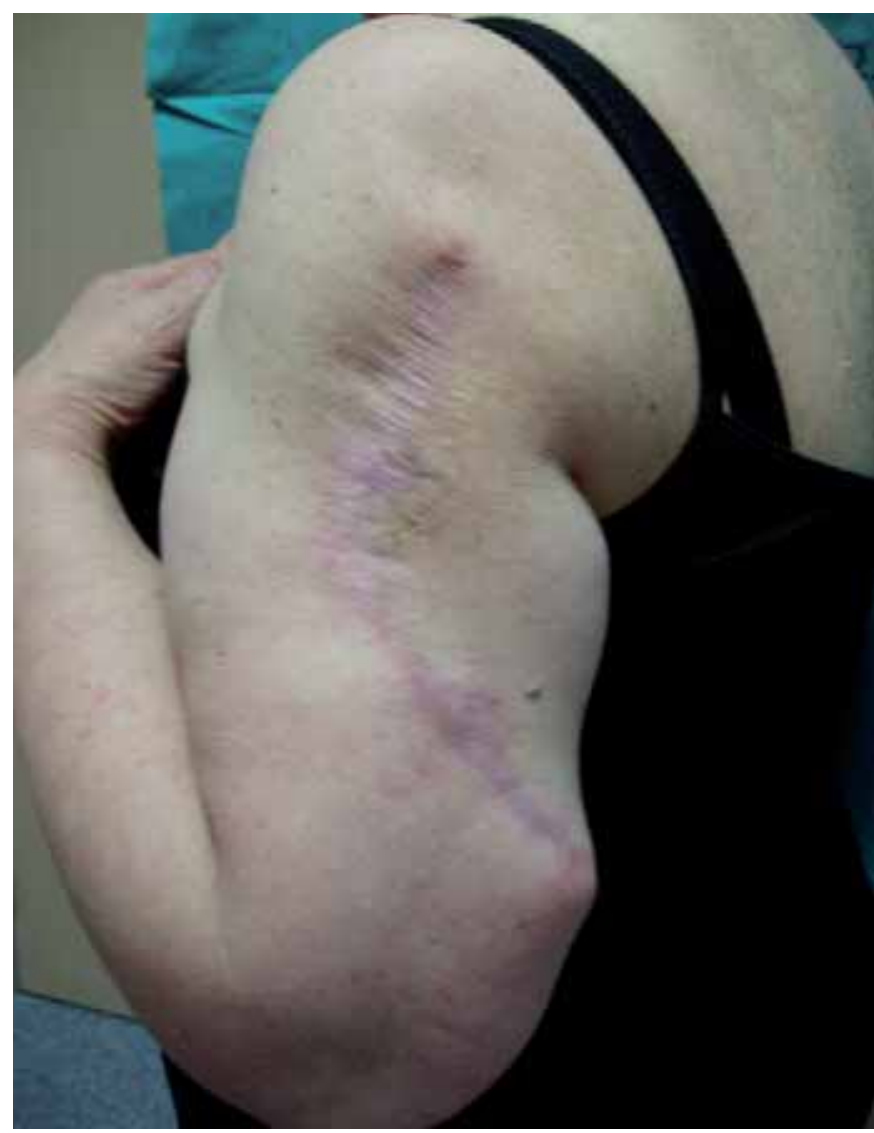

Fig. 10. Resultado postoperatorio a los 2 años.

tipotencialidad de estas células explica la presencia de áreas mixoides y condroides (13).

El siringoma condroide maligno tiene un curso clínico impredecible, que puede cursar curación, recurrencias locales (hasta el 50\% de los casos según las series) (6), metástasis locales y a distancia (39\% y $36 \%$ de los casos respectivamente) (6). Las metástasis a distancia suelen localizarse en los ganglios linfáticos, hueso y órganos (pulmón, cerebro...) $(15,16)$ y son más frecuentes en mujeres.

Por tanto, la recomendación frente a este tipo de tumores es un tratamiento quirúrgico precoz y agresivo, que consiste en la extirpación tumoral completa con márgenes amplios y el seguimiento cercano para detectar las recurrencias locales y las lesiones metastásicas (6). Hirsch y Helwig observaron que las recurrencias solo ocurren con exéresis incompletas, pero a pesar de esto recomiendan realizar seguimiento, y más si el tumor es maligno (4).

La radioterapia o la quimioterapia pueden ser útiles en el siringoma recurrente o si hay atipia citológica, pero no hay evidencia de la efectividad de ambas en la disminución de la masa tumoral; sin embargo sí hay evidencia de la efectividad del tratamiento sobre las lesiones metastáticas.

\section{Conclusiones}

El siringoma condroide maligno es un tumor cutáneo muy infrecuente pero que debemos tener en cuenta en el diagnóstico diferencial de los tumores cutáneos. Su pronóstico es bueno cuando la exéresis es completa, aunque es necesario realizar un seguimiento completo de los pacientes.

\section{Dirección del autor}

Dra. M $M^{\text {a }}$ Pilar Casteleiro Roca

C/Méndez Núñez n³4 - 1º. Narón 15407. Coruña. España.

e-mail: mpcasteleiro @yahoo.es

Bibliografía

1. John TH Mandeville: "Cutaneous Benign Mixed Tumour (chondroid siringoma) of the Eyelid; Clinical Presentation and Management". Ophthalmix Plastic and Reconstructive Surgery 2004,20 (2): 110 .

2. Lever WF, Schaumburg-Lever G.: "Histopathology of the Skin". $6^{\text {th }}$ ed. Philadelphia: JB Lippincott; 1983, Pp:560-2.

3. Billroth T.: "Beobachtungen uber geschwulste der speicheldrusen". Virchows Arch Pathol Anat 1859;17:357.

4. Hirsch P, Helwig EB.: "Chondroid siringoma: mixed tumour of the skin, salivary gland type". Arch Dermatol. 1961; 84:835.

5. Moreno Giménez J.C., Ortega Medina I., Pérez Bernal A.M., Galera Davidson H., Camacho Martínez F.: " Siringoma condroide: aspectos clínico-patológicos”. Med Cut. I. L. A. Vol. XI/1983: 159 .

6. Takahashi H., Ishiko A., Kobayashi M., Tanikawa A., Takasu H., Tanaka M.: "Malignant chondroid siringoma with bone invasion: a case report and review of the literature" Am J Dermatopathol 2004, 26 (5): 403

7. Toback TG, Toback CJ.: "Chondroid siringoma: a review of the literature and case report". J Am Pediatr Med Assoc 1989; 79:563.

8. Gupta S, Kumar A, Padmanabhan A, Khanna S.: "Malignant chondroid siringoma: a clinicopathological study and a collective review". J Surg Oncol 1982;20:139.

9. Chao PZ, Lee FP.: "Pleomorphic adenoma (chondroid siringoma) on the face" Otolaryngol Head Neck Surg. 2004: 130.

10. Kunikane $\mathbf{H}$, Ishikura $\mathbf{H}$, Yamaguchi $\mathbf{J}$, et al.: "Chondroid siringoma (mixed tumor of the skin)". Acta Pathol Jpn 1987; 37(4): 65.

11. Argenzy ZB, Balogh K, Goeken JA.: "Inmunohistochemical characterization of chondroid syringomas". Am J Clin Pathol. 1988;90:662.

12. Kaushik V., Bhalla RK., Nicholson C., de Carpentier JP.: "The chondroid siringoma: report of a case arising from the external auditory canal". Eur Arch Otorhinolaryngol 2005, 262:868.

13. Nigam S., Dhingra KK., Gulati A: "Ectomesenchymal chondromyxoid tumour of the hard palate: a case report" J Oral Pathol Med 2006, 35: 126.

14. Botha JBC, Kahn LB: "Aggressive chondroid siringoma". Arch Dermatol 1978, 114:954.

15. Matz LR, McCully Dj, Stokes B.: "Metastasising chondroid siringoma: a case report". Pathology 1969, 1: 77.

16. Ishimura $\mathrm{E}$, Iwamoto $\mathrm{H}$, Kobashi Y, Yamabe H, Ichijima K.: "Malignant chondroid syringoma. Report of a case with widespread metastasis and review of pertinent literature". Cancer 1983, 52: 1966. 